\title{
BMJ Open Biopsychosocial model of resilience in young adults with multiple sclerosis (BPS-ARMS): an observational study protocol exploring psychological reactions early after diagnosis
}

\author{
Alberto Gajofatto, Valeria Donisi, Isolde Martina Busch, Francesca Gobbin, \\ Elena Butturini, Massimiliano Calabrese, Alessandra Carcereri de Prati, \\ Paola Cesari, Lidia Del Piccolo, Massimo Donadelli, Paolo Fabene, Stefania Fochi, \\ Macarena Gomez-Lira, Roberta Magliozzi, Giovanni Malerba, Raffaella Mariotti, \\ Sofia Mariotto, Chiara Milanese, Maria Grazia Romanelli, Andrea Sbarbati, \\ Federico Schena, Maria Angela Mazzi, Michela Rimondini
}

To cite: Gajofatto A, Donisi V, Busch IM, et al. Biopsychosocial model of resilience in young adults with multiple sclerosis (BPS-ARMS): an observational study protocol exploring psychological reactions early after diagnosis. BMJ Open 2019;9:e030469. doi:10.1136/ bmjopen-2019-030469

- Prepublication history for this paper is available online. To view these files, please visit the journal online http://dx.doi. org/10.1136/bmjopen-2019030469).

Received 15 March 2019 Revised 06 July 2019 Accepted 12 July 2019

Check for updates

C Author(s) (or their employer(s)) 2019. Re-use permitted under CC BY-NC. No commercial re-use. See rights and permissions. Published by BMJ.

Department of Neuroscience, Biomedicine and Movement Sciences, University of Verona, Verona, Italy

Correspondence to

Professor Michela Rimondini; michela.rimondini@univr.it

\section{ABSTRACT}

Introduction Multiple sclerosis (MS), the most common neurological disease causing disability in young adults, is widely recognised as a major stress factor. Studies have shown that the first years after the diagnosis are distressing in terms of adjustment to the disease and that MS negatively affects patients' psychological well-being, quality of life $(\mathrm{Q} o \mathrm{~L})$ and social functioning. However, the links between disease-specific variables at diagnosis, resilience and psychological adjustment of patients with MS remain largely unexplored, especially in adolescents and young adults. This observational study aims to fill the gap of knowledge on biopsychosocial characteristics and resilience of young adults with MS to evaluate the relationship among these variables and to develop a biopsychosocial model of resilience.

Methods and analysis Biological and clinical characteristics of young adults newly diagnosed with MS will be investigated by collecting clinical information, performing neurological examinations, MRI and analysing cerebrospinal fluid and blood biomarkers (eg, measures of inflammation), body composition, gut microbiota and movement/perceptual markers. Psychosocial characteristics (eg, psychological distress, coping strategies), QoL, psychological well-being and resilience will be assessed by self-report questionnaires.

Comparative statistics (ie, analysis of variance or unpaired samples t-test, correlation and regression analyses) will be applied to evaluate the relationship among biological, psychological and social factors. The results are expected to allow a comprehensive understanding of the determinants of resilience in young patients with MS and to inform resilience interventions, tailored to young patients' specific needs, aiming to reduce the risk of maladaptive reactions to the disease and to improve psychological well-being and QoL.

Ethics and dissemination The study has been approved by the Verona University Hospital Ethics Committee (approval number: 2029CESC). The findings will be
Strengths and limitations of this study

- This study provides a novel research approach to patients with multiple scleroris (MS), exploring the relationship between disease-specific variables, resilience and psychological adjustment after diagnosis.

- Study results are expected to expand the understanding of resilience determinants in young adults with MS and to inform resilience interventions with the potential of improving patients' quality of life, well-being and adherence to MS care strategies.

- The present study has the advantage of recruiting patients with MS at a young age, early after diagnosis and before disease-modifying therapy initiation, therefore limiting the impact of long-standing disease and treatment on psychosocial characteristics.

- The methodological approach is multidisciplinary and it favours an integrated vision of MS presentation from different perspectives.

- Study limitations include the cross-sectional design preventing prospective evaluation of the variables of interest and the scarcity of preliminary data to support the expected connections between biological and psychological characteristics of patients with MS.

disseminated through scientific publications in peerreviewed journals, conference presentations, social media and specific websites.

Trial registration number ClinicalTrials.gov (NCT03825055).

\section{INTRODUCTION}

Multiple sclerosis (MS) is a chronic inflammatory disorder of the central nervous system pathologically characterised by the presence 
of scattered areas of demyelination and axonal loss in the brain and spinal cord. ${ }^{1}$ Depending on the location and the accrual rate of such lesions, patients with MS experience episodes of diverse neurological symptoms, which are typically reversible in the early phase of the disease but tend to be replaced by a relentless progression of disability in more advanced stages. ${ }^{2}$ Being diagnosed with MS represents a major life event influencing individual functioning and emotional well-being. For many patients, the first years after the diagnosis are particularly distressing in terms of adjustment to the disease, may negatively affect quality of life (QoL) and social functioning and may lead to psychological symptoms, such as distress, anxiety and depression. ${ }^{3-6}$ However, the findings regarding psychopathology among adolescents and young adults with MS are ambiguous with two studies reporting similar levels of depression and anxiety between young patients and their healthy peers. ${ }^{78}$

Since MS is usually diagnosed in young adults (20-40 years old) and increasingly in adolescents, ${ }^{9}{ }^{10}$ it is important to consider that the personal development, which is crucial for young adults, may be affected and overshadowed by the diagnosis. Various areas of life, such as education, work, relationships and social participation, need to be adjusted to gain a new sense of coherence that necessarily has to include MS. ${ }^{71-13}$ Studies examining patients' adjustments to MS demonstrated a wide range of possible reactions to the diagnosis and different levels of resilience. Indeed, evidence indicates that higher levels of resilience are related to lower psychopathology in adults newly diagnosed with MS. ${ }^{14}$ Considering the association between social support and mental health outcomes, a recent longitudinal study ${ }^{15}$ highlighted the mediating role of resilience in this relationship in patients with MS. However, the amount of received social support may depend on the functional limitations associated with MS, which may reduce participation in social life. ${ }^{16} 17$ As Southwick et $a l^{18}$ stated, 'determinants of resilience include a host of biological, psychological, social, and cultural factors that interact with one another to determine how one responds to stressful experiences'.

Personality traits have been studied as individual factors that can account for differences in health, well-being and overall QoL in MS. While some authors did not find different personality traits between patients with MS and healthy subjects, others described 'Type D personality', defined by high neuroticism, low extraversion, sometimes low conscientiousness, and, to a lesser extent, low agreeableness, in this category of patients. ${ }^{19-22}$ In a sample of 119 adults with MS, trait mindfulness was linked to adaptive coping styles and increased resilience coping skills and higher resilience. ${ }^{23}$

Furthermore, literature has highlighted the important role of coping strategies in the life of patients with MS. A systematic review by Keramat Kar et $a l^{24}$ demonstrated that patients with MS, especially in the beginning, apply mainly emotional and avoidance coping strategies and tend to use, compared with healthy subjects, fewer active coping strategies. Even if coping strategies of patients with MS have been widely investigated, also in relation to psychological constructs, such as personality traits, cognitive impairment and QoL, ${ }^{25}$ the impact of disease-related variables on the coping strategies of patients with MS and, in particular, on resilience mechanisms, remains largely underexamined. Only one systematic review ${ }^{26}$ showed a consistent association between coping strategies of patients diagnosed with life-threatening diseases, such as cancer, HIV/AIDS or MS and post-traumatic growth, which might be considered a dimension of resilience. ${ }^{27}$

Illness perception, defined as the pattern of cognitive representations created by patients facing a new health threat, ${ }^{28}$ is considered another factor potentially influencing the adjustment to MS. ${ }^{29}{ }^{30}$ These beliefs can influence coping behaviour, since negative illness perceptions are related to worse adjustment outcomes, slower recovery and increased future disability. ${ }^{28}$ For this reason, illness perception may influence the ability to be resilient facing the new MS diagnosis.

There is solid evidence that stressful life events are a triggering factor for MS relapses. ${ }^{31}{ }^{32}$ While some earlier studies have also suggested an association between distress and the onset of MS, ${ }^{33} 34$ a recent article could not confirm this finding. ${ }^{21}$

Further, Benedict and colleagues ${ }^{19}$ conducted a study on 120 subjects with MS of any duration to assess the association between clinical and psychological factors and QoL. The findings indicated that self-reported health-related QoL was most strongly predicted by measures of depression, whereas employment status was primarily predicted by measures of cognitive function.

Also, sociodemographic factors, such as gender, age, education and occupational status, need to be considered when studying differences in adapting to MS and resilience, as done in a recent review of coping with $\mathrm{MS}^{24}$

None of the validated diagnostic and prognostic tools that are used in MS clinical practice (eg, type of clinical onset, brain and spinal cord MRI characteristics, IgG oligoclonal bands in the cerebrospinal fluid (CSF) and evoked potentials) have been specifically explored as possible modifiers of patients' psychological characteristics or vice versa. However, it could be hypothesised that clinical and paraclinical features of MS directly or indirectly influence patient's resilience strategies, by interfering with psychological adjustment or other mechanisms still to be investigated in the MS population. ${ }^{35}$ In addition, investigational biomarkers -including neurofilament light chain and cytokines in CSF / serum, ${ }^{3637}$ microRNA expression in cells and tissues, ${ }^{38}{ }^{39}$ oxidative stress markers in body fluids ${ }^{40}{ }^{41}$ gut microbiota composition, ${ }^{42}{ }^{43}$ body composition $^{44} 45$ and neurophysiological markers of motor-perception interactions ${ }^{46}{ }^{47}$-are linked to diverse pathophysiological processes of MS, which could have direct or indirect connections to psychological factors, coping strategies, well-being and ultimately QoL.

A comprehensive understanding of the concept of resilience in the MS field and of related factors, which may 
enhance or reduce resilience, are essential for improving patients' well-being. The role of disability-specific, biological, psychological and social factors should be explored adopting the well-established biopsychosocial model of Engel. ${ }^{48}$ Recently, Black and Dorstyn ${ }^{16}$ tested a biopsychosocial model of resilience in 196 adults with MS. While positive affect and self-efficacy showed a direct effect on resilience, fatigue severity, physical dependence and social support had an indirect effect. However, the authors acknowledged that more than half of the variance could not be explained by the identified variables and therefore suggested to explore in future studies other key elements of resilience from a biopsychosocial perspective.

There is only scant research about resilience in patients with MS and its influencing factors, particularly in adolescents and young adults. Up to now, to our knowledge, only one Italian study ${ }^{7}$ assessed resilience in adolescents and young adults newly diagnosed with MS. Moreover, rarely ${ }^{16}$ have been biological, psychological and social factors potentially associated with resilience explored in a holistic biopsychosocial model.

Thus, investigating resilience in this population is innovative and extremely relevant for the following reasons: (1) youth is a critical period for the individual's future development and well-being; (2) MS is the most common neurological disease causing disability in young people ${ }^{49}$ and increasingly recognised in adolescents; (3) early interventions, focusing on young patients' resilience, are crucially needed to prevent a worsening of potential psychological problems later on in life and to improve psychological well-being and QoL.

\section{OBJECTIVES}

Drawing on the above outlined literature, the aims of the biopsychosocial characteristics and resilience of young adults with MS (BPS-ARMS) are threefold:

Research objective 1: To assess the biological, psychological and social characteristics of a sample of young adults newly diagnosed with MS.

Research objective 2: To explore the resilience strategies and QoL in a sample of young adults newly diagnosed with MS.

Research objective 3: To investigate the association between biopsychosocial characteristics and resilience to develop a biopsychosocial model of resilience in young adults newly diagnosed with MS.

\section{METHODS}

\section{Study design}

The BPS-ARMS project has an observational design. It will last for 3 years, following two main consequential phases (start date: 26 February 2019, planned end date: 26 August 2021). In the first phase, biopsychosocial factors will be collected and resilience strategies and QoL assessed. In the second one, the relationship between biopsychosocial factors and resilience will be explored and consequently a

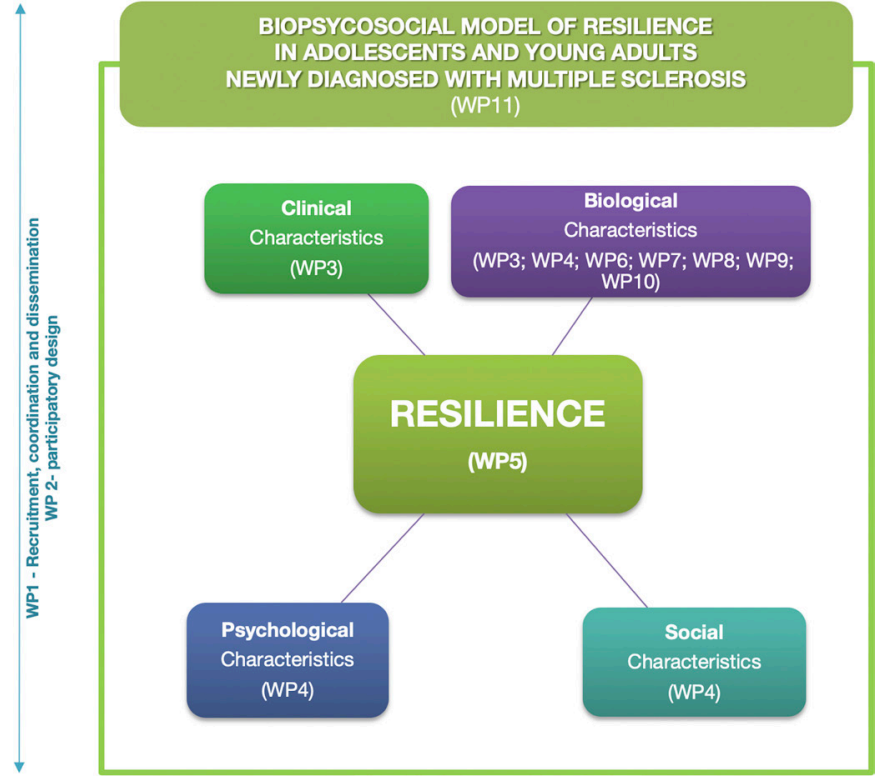

Figure 1 Overview of the BPS-ARMS work packages. BPSARMS, biopsychosocial characteristics and resilience of young adults with MS.

biopsychosocial model of resilience in young adults newly diagnosed with MS will be developed. Eleven specific work packages (WP) will be accomplished during these two phases (figure 1).

For each WP, different actions will be performed according to the flowchart (figure 2). WP1 actions include recruitment, coordination and dissemination of the results. WP2 aims to achieve an effective, constant collaboration between researchers, patients and healthcare providers (participatory design). WP3 is dedicated to the screening phase for the eligibility of patients and collection of clinical and MRI characteristics of young patients and analysis of protein biomarkers and 24OH-Cholesterol. WP4 aims to collect the psychosocial characteristics of young patients. WP6, 7, 8, 9 and 10 aim to collect all the biological variables not included in WP3.

Research actions of WP3 and WP6-10 are summarised in table 1. WP4 procedures are reported in table 2. Exploration of resilience and QoL will be performed in WP5 and, considering the results of the previous WPs, WP11 procedures will allow developing the biopsychosocial model of resilience.

\section{Sample and setting}

The BPS-ARMS project will be implemented in the Multiple Sclerosis Regional Center of the Azienda Ospedaliera Universitaria Integrata of Verona-Borgo Roma Hospital (Veneto Region, Italy-Hub for Verona Province).

Patients will be enrolled according to the following inclusion criteria: age range 18-40 years; MS diagnosis in the 2 years prior to study inclusion, according to the revised McDonald Criteria ${ }^{50}$; MRI of the brain in the 6 months prior to or within 1 month after screening visit, according to the protocol described below; Italian speakers. 


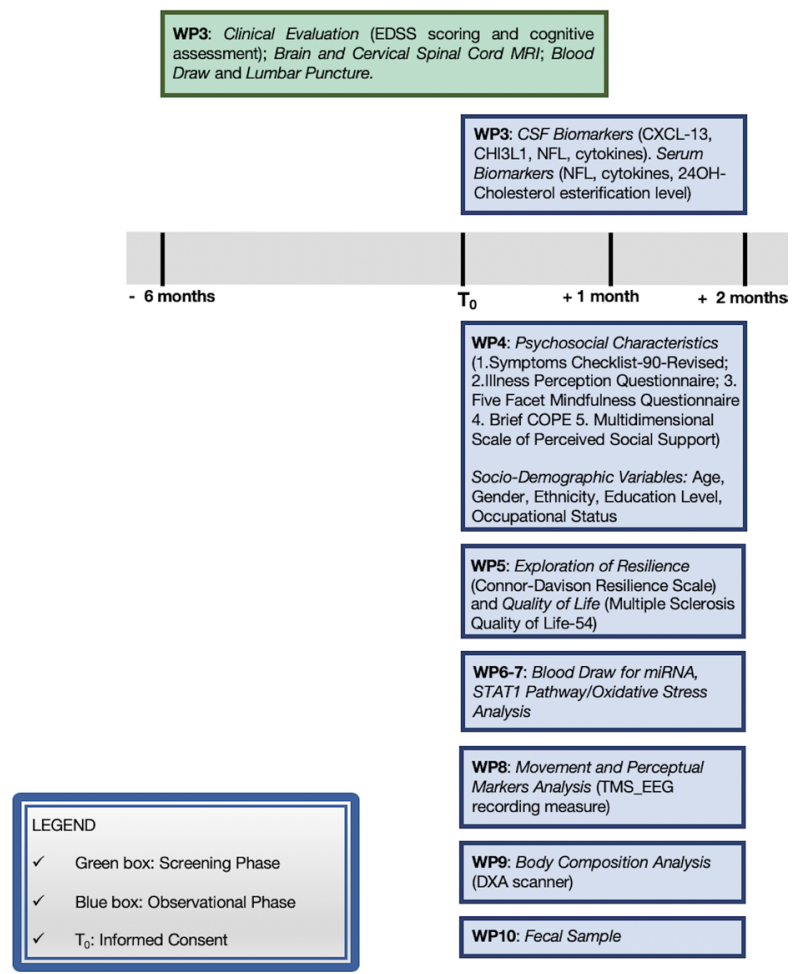

Figure 2 Flowchart of data collection according to each WP. COPE, Coping Orientation to the Problems Experienced; CSF, cerebrospinal fluid; DXA, dual-energy X-ray absorptiometry; EDSS, Expanded Disability Status Scale; EEG, electroencephalography; miRNA, microRNA; NFL, neurofilament light chain; TMS, transcranial magnetic stimulation; WP, work package.

We will exclude patients with: clinically relevant cognitive deficits as evaluated by the treating neurologist; treatment with any disease-modifying therapy for MS at inclusion and by completion of study procedures (maximum 2 months from consent); steroids administration up to 30 days prior to inclusion is allowed.

The effective sample size, required to allow the regression models to be reliably estimated, is $150-180$ patients. On the basis of the setting catchment area (ie, 1800 patients with MS annually for Verona province), we estimate that the required numerosity of eligible patients will be reached in a time period of 30 months.

\section{Recruitment procedures}

Eligible patients will be consecutively enrolled by the neurologists and residents working at the MS Center of Borgo Roma Hospital, Verona. During the first visit at the MS Center, the neurologists/residents will explain the study to eligible patients, and the consent form will be signed. During this visit, sociodemographic and clinical information will be collected, according to routine clinical practice. Within 1 month from consent, patients will start a screening phase, which is part of the typical diagnostic work-up of patients with suspected MS (WP3). After screening completion, fulfilment of inclusion criteria and absence of exclusion criteria, patients will be enrolled or excluded from the study accordingly. Within 1 month from screening completion, patients included in the study will undergo a blood draw from a peripheral vein at the MS Center, and a faecal sample will be collected (WP 3, 6, 7 and 10). During the same visit, a psychological battery of tests will be performed (questionnaires for both WP4 and WP5). Within 1 month from screening completion, patients can decide to be involved in additional study procedures, regarding the analysis of movement and perceptual markers as well as body composition as measured by dual-energy X-ray absorptiometry (WP8 and WP9) at the dedicated laboratories of the University of Verona. All the aforementioned procedures will

Table 1 Summary of the clinical and biological variables collected in WPs $3,6,7,8,9,10$

Work

package Description

WP3 Recruitment and screening phase; collection of clinical, MRI and CSF data of patients; analysis of NFL in serum/CSF by Simoa Quanterix; analysis of inflammatory cytokine panel in serum/CSF by Bioplex, BioRad ${ }^{37}$; analysis of $24 \mathrm{OH}-\mathrm{Cholesterol} \mathrm{esterification}$ level in serum ${ }^{61}$

WP6 Analysis of microRNA levels in exosomes from serum samples. Candidate miRNAs to be analysed (miR-15b-5p, miR-374a-5p miR-342-3p, miR-30b-5p, miR-223-3p, miR-433-3p, miR-432-5p, miR-23a-3p, miR-485-3p and let-7i) have been selected from previous studies indicating them as potential biomarkers for MS. ${ }^{38} 39$

\begin{tabular}{|c|c|}
\hline WP7 & $\begin{array}{l}\text { Oxidative stress markers analysis. The concentration of glutathione and glutathione disulphide (GSH/GSSG), of nitrite/nitrate } \\
\left(\mathrm{NO}_{2}^{-} / \mathrm{NO}_{3}^{-}\right) \text {as well as levels of lipid peroxidation and of oxidative post-translation modification of proteins will be evaluated in } \\
\text { plasma. The activation of STAT1 signalling (phosphoTyr701-STAT1/STAT1 ratio and levels of S-glutathionylated STAT1) will be } \\
\text { analysed in PBMC. }{ }^{40} 62\end{array}$ \\
\hline WP8 & $\begin{array}{l}\text { Movement and perceptual markers analysis. }{ }^{46}{ }^{47} \text { Upper extremities motor evoked potentials obtained by transcranial magnetic } \\
\text { stimulation in two tasks (ie, rest and motor imagery) }\end{array}$ \\
\hline WP9 & $\begin{array}{l}\text { Body composition analysis. }{ }^{44}{ }^{45} \text { Bone mineral content, fat-free soft tissue mass, fat mass and percentage of fat mass will be } \\
\text { measured by dual-energy X-ray absorptiometry }\end{array}$ \\
\hline WP10 & $\begin{array}{l}\text { Gut microbiota composition. }{ }^{42}{ }^{43} \mathrm{~A} \text { faecal sample will be collected at enrollment and analysed to assess microbiome composition } \\
\text { using the percentage of bacterial subgroups at the genus level and protein concentration resulting from metaproteomics } \\
\text { experiments. }\end{array}$ \\
\hline
\end{tabular}

CSF, cerebrospinal fluid; MS, multiple sclerosis; NFL, neurofilament light chain; PBMC, peripheral blood mononuclear cell; WP, work package. 
Table 2 Summary of the psychological variables and self-report questionnaires (WP4)

\begin{tabular}{|c|c|c|}
\hline Measure & Questionnaire & Description of the questionnaires and variables \\
\hline Symptoms of psychopathology & SCL-90-R ${ }^{63}$ & $\begin{array}{l}\text { SCL-90-R is a } 90 \text {-item questionnaire, designed to evaluate, } \\
\text { on a 5-point Likert scale. A broad range of psychological } \\
\text { problems and symptoms of psychopathology. The primary } \\
\text { assessed dimensions are somatisation, obsessive- } \\
\text { compulsive, interpersonal sensitivity, depression, anxiety, } \\
\text { hostility, phobic anxiety, paranoid ideation and psychoticism. }\end{array}$ \\
\hline Mindfulness traits & $\begin{array}{l}\text { The Italian short form of FFMQ-SF } \\
65\end{array}$ & $\begin{array}{l}\text { The FFMQ-SF with } 24 \text { items is used to assess major } \\
\text { aspects of mindfulness skills: observing, describing, acting } \\
\text { with awareness, non-judging and non-reactivity. Items are } \\
\text { measured on a 5-point Likert scale. }\end{array}$ \\
\hline Coping strategies & The Brief COPE ${ }^{66-68}$ & $\begin{array}{l}\text { The Brief COPE includes } 28 \text { items, organised in } 14 \text { subscales, } \\
\text { each composed of } 2 \text { items. Each item is evaluated on a } \\
\text { 4-point scale from } 1 \text { to } 4 \text {. }\end{array}$ \\
\hline Illness perception & The Brief IPQ ${ }^{29} 69-71$ & $\begin{array}{l}\text { The Brief IPQ-R is a 9-item instrument developed to provide } \\
\text { a quantitative measurement of the components of illness } \\
\text { representations, evaluated on a 5-point Likert scale. }\end{array}$ \\
\hline Social support & The Italian version of MSPSS ${ }^{745}$ & $\begin{array}{l}\text { The MSPSS, a 12-item measure of social support, measures } \\
\text { on a } 6 \text {-point Likert scale the level of perceived social support } \\
\text { of various sources (eg, family, friends and significant others). }\end{array}$ \\
\hline
\end{tabular}

COPE, Coping Orientation to the Problems Experienced; FFMQ-SF, Five Facet Mindfulness Questionnaire Short Form; IPQ, Illness Perception Questionnaire; MSPSS, Multidimensional Scale of Perceived Social Support; PSI, Psychosocial Index; SCL-90-R, Symptoms Checklist 90 items Revised.

be completed within 2 months from informed consent to guarantee timely access to the more appropriated MS treatment for each patient as discussed between the treating neurologist and the patient according to clinical practice. Study procedures will not influence treatment decisions.

\section{Variables and procedures}

The primary endpoint of the study is the resilience of patients with MS, assessed using the Connor-Davison Resilience Scale (CD-RISC). ${ }^{51}$ This instrument is specifically designed to examine resilience features in adolescents and adults. The CD-RISC is composed of 25 items and evaluated on a 5-point Likert scale (ranging from 0 'not true at all' to 4 'true nearly all of the time'), with higher scores reflecting higher levels of resilience. This instrument has been selected because of its good psychometric properties. ${ }^{51}$ Further, it showed the highest quality in a methodological review ${ }^{52}$ evaluating 15 resilience questionnaires. The psychometric properties of the Italian version of the 25-item CD-RISC will be described by using item response theory analysis on our sample of patients with MS. To validate this scale in the Italian language, a confirmatory factor analysis (CFA) will be performed. Furthermore, this questionnaire has already been used with patients with MS ${ }^{15162353}$ and translated into Italian.

The secondary endpoint is QoL, assessed using the Italian version of the Multiple Sclerosis Quality of Life-54 (MSQoL54) ${ }^{54}$ The MSQOL-54 is a multidimensional health-related QoL measure that combines both generic and MS-specific items, such as fatigue and cognitive function. ${ }^{54-56}$ The instrument generates 12 scores (ie, physical function, role limitations-physical, role limitations-emotional, pain, emotional well-being, energy, health perceptions, social function, cognitive function, health distress, overall $\mathrm{QoL}$ and sexual function) along with two summary scores (ie, physical health and mental health) derived from a weighted combination of scale scores. There are also two single-item measures: satisfaction with sexual function and change in health. The Italian version of MSQOL-54 is easy to administer and well accepted by patients.

All variables collected in the WPs 3, 4 and 6-10 (eg, clinical, psychosocial, MRI and laboratory data) are used to describe the sample and considered as potential predictors of resilience through Exploratory Factor Analysis (EFA).

The following sociodemographic variables will be collected at the recruitment for each patient through a case report form: age, gender, ethnicity, education level and occupational status.

Table 1 recaps the clinical and biological variables for all WPs, with the exception of WP4, for which table 2 describes measures and the questionnaires used to collect the psychological measures. For the variables collected in WPs 3 and 6-10, preliminary measurements, obtained on biological samples of healthy subjects in the laboratories of the research team, will be used as the qualitative reference. This will allow setting up the methods for biomarkers analysis for each WP. 
Patient and public involvement statement. Participatory design: research partnership with patients and associations

Patients and the public were first involved in the conclusive phase of protocol preparation during a meeting with Verona representatives of the Italian Multiple Sclerosis Association (AISM) and during a public event organised by the University of Verona in September 2018. Psychological variables and outcome measures were developed based on the response to a preliminary survey that was conducted in a sample of patients with MS attending the Verona MS centre in 2017.

The Verona section of AISM will be involved in the recruitment process through disseminating information to patients and caregivers regarding the study. A patients advisory board (PAB) composed by young patients with MS and representatives of AISM will be established. The recruitment will be performed at the beginning of the project in the clinical centres and MS associations of patients and relatives. The PAB will collaborate and supervise all the research phases (including the discussion, interpretation and dissemination of results) through regular meetings and consultations.

The participatory design of the study (WP2) will increase the ecological validity of our results and improve trust and collaboration among the MS stakeholders as a potential starting point for future research and care planning.

\section{Planned analysis}

An integrated database will be created and checked for completeness across all the collected biopsychosocial variables. It will be possible to merge the databases (ie, of WP3, WP4 and so on) using the unique study subject identifier. The statistical power analysis used to identify the sample size is based on the number of subjects per variable (SPV) approach. ${ }^{5758}$ The literature indicates 10 as the minimum value of SPV. In the present study, we have set SPV at 15, given that we analyse one relevant variable (ie, indicator) for each WP (ie, WP3-WP10) and five clinical and sociodemographic characteristics (sex, age, education level, Expanded Disability Status Scale score and the number of T2 lesions on brain MRI). These characteristics will be analysed a priori in the regression models due to the relevance for the resilience of patients with MS.

Since the relationship between resilience and biological variables has been scarcely investigated in the literature, the handling of missing data could be problematic, particularly in case of missing not at random. ${ }^{59}$ For this reason, only the variables with an amount of missing data $<10 \%$ will be included in the analysis so that the results can be considered robust and accurate.

To identify the indicator for each WP to be included in the final biopsychosocial model of resilience, an EFA will be performed using each WP variable measured as factors. The first factor (ie, the one explaining most of the variability) will be selected as the indicator for the specific WP. Applying generalised linear regression models, we will preliminarily explore (1) the relationship among the different WP indicators and (2) the relationship between each WP indicator and resilience. The selected WP indicators will be jointly explored using multivariable models to quantify the main relationships. The results of the regression analyses will lead to the development of a biopsychosocial model of resilience. Given the paucity of evidence in this field, we will proceed gradually in the design of the model, applying different statistical techniques according to the critical issues that will potentially emerge from the preliminary descriptive analysis. If the main assumptions of Structural Equation Models (ie, the sample size of groups, the normality of frequency distribution of the variables of interest, the absence of outliers) will be satisfied, it will be possible to perform also CFAs. This method will allow the goodness of fit of our biopsychosocial model to be assessed.

The same procedure will be applied at the secondary endpoint of the study to build a biopsychosocial model of QoL. Statistical analyses will be carried out using Stata V.15. ${ }^{60}$

\section{ETHICS AND DISSEMINATION \\ Ethics and safety}

The project will be conducted according to the approved protocol. All data collected during the project will be handled fairly, and the databases will remain strictly confidential, anonymised, password protected and stored in locked and secured facilities at the Department of Neuroscience, Biomedicine and Movement Sciences, University of Verona.

In detail, patients who sign the informed consent form and comply with all of the inclusion criteria will be assigned a unique study subject identifier (ie, a pseudonym or alias). Only the authorised study personnel will have access to the matching between the subject identifier and patient identity. The study subject identifier will remain the same during the whole study and will allow the proper handling of the patients' data and samples without using sensitive personal identifiers. Data will be collected in a dedicated case report form and entered into an electronic database for analysis.

The collected biological samples will be stored at the Neuropathology Laboratory of the University Hospital of Verona for 10 years and may be used for additional research conducted at the University of Verona and related to the present study. Following the completion of the study, leftover samples sent to the laboratories of the "Centro Interuniversitario di Ricerca sui Peptidi Bioattivi” (CIRPEB, Research Centre on Bioactive Peptides), Napoli (see WP3) will be destroyed after analysis.

\section{Dissemination plan}

A dissemination plan will be established in the first phase of the project in agreement with all the involved researchers during a dedicated meeting. The dissemination target will be different stakeholders (eg, other researchers, patients, MS representatives and associations, healthcare professionals, the general public). Each dissemination product will be tailored to these different 
target groups in terms of objectives, methods, timeline and language (eg, youth-friendly language and format). Facebook and specific websites will be used to disseminate the materials of the project. The use of these social media channels will allow a broader dissemination of the project results. The scientific community will be reached through scientific publications in national and international preferably open access journals and presentations at national and international scientific conferences. Items for dissemination in any format including publications will not contain any information that could lead to the identification of the patients.

Contributors All the authors were involved in planning and conceptualising the project providing their specific contribution according to the different disciplines. AG, MR, VD, FG, EB, MC, ACDP, PC, MD, PF, SF, MGL, R Magliozzi, GM, R Marriotti, $\mathrm{SM}, \mathrm{CM}, \mathrm{MGR}$ and $\mathrm{AS}$ were involved in the acquisition of data. AG, MR, MAM, VD, IMB, FG, EB, MC, ACDP, PC, MD, PF, SF, MGL, R Magliozzi, GM, R Marriotti, SM, CM, MGR, LDP, FS and AS were involved in the analysis and interpretation of the data. $A G, V D, I M B$ and MR drafted the manuscript. MAM provided her expertise on the methodological elements. All authors reviewed and approved the final manuscript. In their role as team leaders, MR and AG supervised the entire project. In their role as project managers, LDP and FS coordinated the general organisation and institutional aspects of the project.

Funding Italian Ministry of Research and University (MIUR) 5-year special funding to strengthen and enhance the excellence in research and teaching (https:// www.miur.gov.it/dipartimenti-di-eccellenza). The present study has been partially supported by this programme.

Competing interests None declared.

Patient and public involvement statement Patients or the public were involved in our work.

\section{Patient consent for publication Not required.}

Ethics approval BPS-ARMS has been approved by the Ethical Committee of Verona and Rovigo Province (Azienda Ospedaliera Universitaria Integrata di Verona)—study registration number: Prog. 2029CESC.

Provenance and peer review Not commissioned; externally peer reviewed.

Open access This is an open access article distributed in accordance with the Creative Commons Attribution Non Commercial (CC BY-NC 4.0) license, which permits others to distribute, remix, adapt, build upon this work non-commercially, and license their derivative works on different terms, provided the original work is properly cited, appropriate credit is given, any changes made indicated, and the use is non-commercial. See: http://creativecommons.org/licenses/by-nc/4.0/.

\section{REFERENCES}

1. Filippi M, Brück W, Chard D, et al. Association between pathological and MRI findings in multiple sclerosis. Lancet Neurol 2019;18:198-210.

2. Filippi M, Bar-Or A, Piehl F, et al. Author correction: multiple sclerosis. Nat Rev Dis Primers 2018;4.

3. Janssens ACJW, Buljevac D, van Doorn PA, et al. Prediction of anxiety and distress following diagnosis of multiple sclerosis: a twoyear longitudinal study. Mult Scler 2006;12:794-801.

4. Kern S, Schrempf W, Schneider H, et al. Neurological disability, psychological distress, and health-related quality of life in MS patients within the first three years after diagnosis. Mult Scler 2009;15:752-8.

5. Moss-Morris R, Dennison L, Landau S, et al. A randomized controlled trial of cognitive behavioral therapy (CBT) for adjusting to multiple sclerosis (the saMS trial): does CBT work and for whom does it work? J Consult Clin Psychol 2013;81:251-62.

6. Pagnini F, Bosma CM, Phillips D, et al. Symptom changes in multiple sclerosis following psychological interventions: a systematic review. BMC Neurol 2014;14:222.

7. Rainone N, Chiodi A, Lanzillo R, et al. Affective disorders and healthrelated quality of life (HRQoL) in adolescents and young adults with multiple sclerosis (MS): the moderating role of resilience. Qual Life Res 2017;26:727-36.
8. Messmer Uccelli M, Traversa S, Ponzio M. A survey study comparing young adults with MS and healthy controls on self-esteem, selfefficacy, mood and quality of life. J Neurol Sci 2016;368:369-73.

9. Boesen MS, Magyari M, Koch-Henriksen N, et al. Pediatric-Onset multiple sclerosis and other acquired demyelinating syndromes of the central nervous system in Denmark during 1977-2015: a nationwide population-based incidence study. Mult Scler 2018;24:1077-86.

10. Mah JK, Thannhauser JE. Management of multiple sclerosis in adolescents - current treatment options and related adherence issues. Adolesc Health Med Ther 2010;1:31-43.

11. Calandri E, Graziano F, Borghi M, et al. Improving the quality of life and psychological well-being of recently diagnosed multiple sclerosis patients: preliminary evaluation of a group-based cognitive behavioral intervention. Disabil Rehabil 2017;39:1474-81.

12. Kiropoulos LA, Kilpatrick T, Holmes A, et al. A pilot randomized controlled trial of a tailored cognitive behavioural therapy based intervention for depressive symptoms in those newly diagnosed with multiple sclerosis. BMC Psychiatry 2016;16:435.

13. Thomas PW, Thomas S, Hillier C, et al. Psychological interventions for multiple sclerosis. Cochrane Database Syst Rev 2006;6.

14. Tan-Kristanto S, Kiropoulos LA, Resilience KLA. Resilience, selfefficacy, coping styles and depressive and anxiety symptoms in those newly diagnosed with multiple sclerosis. Psychol Health Med 2015;20:635-45.

15. Koelmel E, Hughes AJ, Alschuler KN, et al. Resilience mediates the longitudinal relationships between social support and mental health outcomes in multiple sclerosis. Arch Phys Med Rehabil 2017;98:1139-48.

16. Black R, Dorstyn D. A biopsychosocial model of resilience for multiple sclerosis. J Health Psychol 2015;20:1434-44.

17. Molton IR, Hirsh AT, Smith AE, et al. Age and the role of restricted activities in adjustment to disability-related pain. $J$ Health Psychol 2014;19:1025-34.

18. Southwick SM, Bonanno GA, Masten AS, et al. Resilience definitions, theory, and challenges: interdisciplinary perspectives. Eur $J$ Psychotraumatol 2014;5:25338.

19. Benedict RHB, Wahlig E, Bakshi R, et al. Predicting quality of life in multiple sclerosis: accounting for physical disability, fatigue, cognition, mood disorder, personality, and behavior change. J Neurol Sci 2005;231:29-34.

20. Bsteh G, Monz E, Zamarian L, et al. Combined evaluation of personality, risk and coping in MS patients: A step towards individualized treatment choice - The PeRiCoMS-Study I. J Neurol Sci 2017;376:71-5.

21. Roy S, Drake AS, Eizaguirre MB. Stressful life events and the risk of initial central nervous system demyelination.. Mult Scler J 2017;23:1000-7.

22. Strober LB. Personality in multiple sclerosis (MS): impact on health, psychological well-being, coping, and overall quality of life. Psychol Health Med 2017;22:152-61.

23. Senders A, Bourdette D, Hanes D, et al. Perceived stress in multiple sclerosis: the potential role of mindfulness in health and well-being. $J$ Evid-Based Integr Med 2014;19:104-11.

24. Keramat Kar M, Whitehead L M. Characteristics and correlates of coping with multiple sclerosis: a systematic review. Disabil Rehabil 2017;10:1-15.

25. Goretti B, Portaccio E, Zipoli V, et al. Coping strategies, cognitive impairment, psychological variables and their relationship with quality of life in multiple sclerosis. Neurol Sci 2010;31:227-30.

26. Barskova T, Oesterreich R. Post-Traumatic growth in people living with a serious medical condition and its relations to physical and mental health: a systematic review. Disabil Rehabil 2009;31:1709-33.

27. Silverman AM, Verrall AM, Alschuler KN, et al. Bouncing back again, and again: a qualitative study of resilience in people with multiple sclerosis. Disabil Rehabil 2017;39:14-22.

28. Petrie K, Weinman J. Why illness perceptions matter. Clin Med 2006;6:536-9.

29. Broadbent E, Petrie KJ, Main J, et al. The brief illness perception questionnaire. J Psychosom Res 2006;60:631-7.

30. Moss-Morris R, Dennison L, Yardley L, et al. Protocol for the saMS trial (supportive adjustment for multiple sclerosis): a randomized controlled trial comparing cognitive behavioral therapy to supportive listening for adjustment to multiple sclerosis. BMC Neurol 2009;9:45.

31. Mohr DC, Hart SL, Julian L, et al. Association between stressful life events and exacerbation in multiple sclerosis: a meta-analysis. BMJ $2004 ; 328$.

32. Artemiadis AK, Anagnostouli MC, Alexopoulos EC. Stress as a risk factor for multiple sclerosis onset or relapse: a systematic review. Neuroepidemiology 2011;36:109-20. 
33. Warren S, Greenhill S, Warren KG. Emotional stress and the development of multiple sclerosis: case-control evidence of a relationship. J Chronic Dis 1982;35:821-31.

34. Grant I, Brown GW, Harris T, et al. Severely threatening events and marked life difficulties preceding onset or exacerbation of multiple sclerosis. Journal of Neurology, Neurosurgery \& Psychiatry 1989;52:8-13.

35. Wu G, Feder A, Cohen $\mathrm{H}$, et al. Understanding resilience. Front Behav Neurosci 2013;7:10.

36. Teunissen CE, Malekzadeh A, Leurs C, et al. Body fluid biomarkers for multiple sclerosis--the long road to clinical application. Nat Rev Neurol 2015;11:585-96.

37. Magliozzi R, Howell OW, Nicholas R, et al. Inflammatory intrathecal profiles and cortical damage in multiple sclerosis. Ann Neurol 2018;83:739-55.

38. Ebrahimkhani S, Vafaee F, Young PE, et al. Exosomal microRNA signatures in multiple sclerosis reflect disease status. Sci Rep 2017;7:142-93.

39. Kimura K, Hohjoh H, Yamamura T. The role for exosomal microRNAs in disruption of regulatory $\mathrm{T}$ cell homeostasis in multiple sclerosis. $J$ Exp Neurosci 2018;12:1179069518764892.

40. Fiorini A, Koudriavtseva T, Bucaj E, et al. Involvement of oxidative stress in occurrence of relapses in multiple sclerosis: the spectrum of oxidatively modified serum proteins detected by proteomics and redox proteomics analysis. PLoS One 2013;8:e65184.

41. Gonsette RE. Neurodegeneration in multiple sclerosis: the role of oxidative stress and excitotoxicity. J Neurol Sci 2008;274:48-53.

42. Trott S, King IL. An introduction to the microbiome and MS. Mult Scler 2018:24:53-7.

43. Haase S, Haghikia A, Wilck N, et al. Impacts of microbiome metabolites on immune regulation and autoimmunity. Immunology 2018;154:230-8.

44. Hadgkiss EJ, Jelinek GA, Weiland TJ, et al. The association of diet with quality of life, disability, and relapse rate in an international sample of people with multiple sclerosis. Nutr Neurosci 2015;18:125-36.

45. Jelinek GA, De Livera AM, Marck CH, et al. Associations of lifestyle, medication, and socio-demographic factors with disability in people with multiple sclerosis: an international cross-sectional study. PLOS One 2016;11:e0161701.

46. Jacobs JV, Kasser SL. Effects of dual tasking on the postural performance of people with and without multiple sclerosis: a pilot study. J Neurol 2012;259:1166-76.

47. Tabrizi YM, Mazhari S, Nazari MA, et al. Compromised motor imagery ability in individuals with multiple sclerosis and mild physica disability: an Erp study. Clin Neurol Neurosurg 2013;115:1738-44.

48. Engel $\mathrm{G}$. The need for a new medical model: a challenge for biomedicine. Science 1977;196:129-36.

49. Rolak LA. Multiple sclerosis: it's not the disease you thought it was. Clin Med Res 2003:1:57-60.

50. Thompson AJ, Banwell BL, Barkhof F, et al. Diagnosis of multiple sclerosis: 2017 revisions of the McDonald criteria. Lancet Neurol 2018;17:162-73.

51. Connor KM, Davidson JRT. Development of a new resilience scale: the Connor-Davidson resilience scale (CD-RISC). Depress Anxiety 2003;18:76-82.

52. Windle G, Bennett KM, Noyes J. A methodological review of resilience measurement scales. Health Qual Life Outcomes 2011;9:8

53. Battalio SL, Silverman AM, Ehde DM, et al. Resilience and function in adults with physical disabilities: an observational study. Arch Phys Med Rehabil 2017;98:1158-64.
54. Solari A, Filippini G, Mendozzi L, et al. Validation of Italian multiple sclerosis quality of life 54 questionnaire. Journal of Neurology, Neurosurgery \& Psychiatry 1999;67:158-62.

55. Vickrey BG, Hays RD, Harooni R, et al. A health-related quality of life measure for multiple sclerosis. Qual Life Res 1995;4:187-206.

56. Vickrey BG, Hays RD, Genovese BJ, et al. Comparison of a generic to disease-targeted health-related quality-of-life measures for multiple sclerosis. J Clin Epidemiol 1997;50:557-69.

57. Austin PC, Steyerberg EW. The number of subjects per variable required in linear regression analyses. $J$ Clin Epidemiol 2015:68:627-36.

58. Ogundimu EO, Altman DG, Collins GS. Adequate sample size for developing prediction models is not simply related to events per variable. J Clin Epidemiol 2016;76:175-82.

59. Ibrahim JG, Chu H, Chen M-H. Missing data in clinical studies: issues and methods. JCO 2012;30:3297-303.

60. Stata statistical software: release 152017College Station, TXStataCorp

61. La Marca V, Spagnuolo MS, Cigliano L, et al. The enzyme lecithincholesterol acyltransferase esterifies cerebrosterol and limits the toxic effect of this oxysterol on SH-SY5Y cells. J Neurochem 2014;130:97-108.

62. Giustarini D, Dalle-Donne I, Milzani A, et al. Analysis of GSH and GSSG after derivatization with $N$-ethylmaleimide. Nat Protoc 2013;8:1660-9.

63. Derogatis LR. Symptom Checklist-90-Revised. London, UK: Pearson, 1994.

64. Bohlmeijer E, ten Klooster PM, Fledderus M, et al. Psychometric properties of the five facet mindfulness questionnaire in depressed adults and development of a short form. Assessment 2011;18:308-20.

65. Iani L, Lauriola M, Cafaro V. The assessment of mindfulness skills: the "what" and the "how". Journal of Mental Health 2017;26:1-8. [Epub ahead of print.

66. Carver CS. You want to measure coping but your protocol's too long: consider the brief cope. Int J Behav Med 1997;4:92-100.

67. Carver CS, Scheier MF, Weintraub JK. Assessing coping strategies: a theoretically based approach. J Pers Soc Psychol 1989;56:267-83.

68. Sica C, Magni C, Ghisi M, et al. Uno strumento aggiornato per La misura degli stili di coping: IL coping orientation to the problems Experienced-Nuova Versione Italiana (COPE-NVI). Psicoterapia Cognitiva e Comportamentale 2008;14:27-53.

69. Baum A, Taylor SE, Singer JE. Handbook of psychology and health Vol. IV. Hillsdale: NJ, 1984

70. Petrie KJ, Weinman J. Perceptions of health and illness: current research and applications. The Netherlands: Amsterdam, 1997.

71. Moss-Morris R, Weinman J, Petrie $\mathrm{K}$, et al. The revised illness perception questionnaire (IPQ-R). Psychol Health 2002;17:1-16.

72. Piolanti A, Offidani E, Guidi J, et al. Use of the psychosocial index: a sensitive tool in research and practice. Psychother Psychosom 2016;85:337-45.

73. Sonino N, Fava GA. A simple instrument for assessing stress in clinical practice. Postgrad Med J 1998;74:408-10.

74. Prezza M, Principato MC. La rete e il sostegno sociale. In: Prezza M, Santinello M, eds. Conoscere la comunit . Bologna. Italy: II Mulino, 2002: 193-233.

75. Zimet GD, Dahlem NW, Zimet SG, et al. The multidimensional scale of perceived social support. J Pers Assess 1988;52:30-41. 